AperTO - Archivio Istituzionale Open Access dell'Università di Torino

\title{
Anticoagulant cessation following atrial fibrillation ablation: limits of the ECG-guided approach
}

\section{This is the author's manuscript}

Original Citation:

Availability:

This version is available http://hdl.handle.net/2318/1639843

since 2017-06-29T12:53:52Z

Published version:

DOI:10.1080/14779072.2017.1332993

Terms of use:

Open Access

Anyone can freely access the full text of works made available as "Open Access". Works made available under a Creative Commons license can be used according to the terms and conditions of said license. Use of all other works requires consent of the right holder (author or publisher) if not exempted from copyright protection by the applicable law. 
This is the author's final version of the contribution published as:

Anselmino, Matteo; Rovera, Chiara; Marchetto, Giovanni; Ferraris, Federico; Castagno, Davide; Gaita, Fiorenzo. Anticoagulant cessation following atrial fibrillation ablation: limits of the ECG-guided approach. EXPERT REVIEW OF CARDIOVASCULAR THERAPY. 15 (6) pp: 473-479.

DOI: 10.1080/14779072.2017.1332993

The publisher's version is available at:

https://www.tandfonline.com/doi/pdf/10.1080/14779072.2017.1332993

When citing, please refer to the published version.

Link to this full text:

http://hdl.handle.net/2318/1639843 
Anticoagulant cessation following atrial fibrillation ablation:

limits of the ECG-guided approach.

Word count: 2829 words, 4 figures, and 31 references

Conflicts of interest: none 


\begin{abstract}
Introduction: Long-term cessation of oral anticoagulation (OAC) following successful catheter or surgical ablation of atrial fibrillation (AF) is debated. Usually, in the presence of sinus rhythm at serial ECG recordings, the CHADS2, CHA2DS2VASc, and HAS-BLED scores are adopted to guide decision regarding OAC management.

Areas covered: The safety of OAC cessation in patients without recurrent AF but with historically elevated risk for thromboembolism remains largely unknown. Taking the cue from two clinical cases, we provide an updated summary of the latest evidence regarding how to manage OAC after a successful atrial fibrillation ablation.

Expert commentary: The present clinical perspective suggests that, at least within patients with severely enlarged left atrium, previous cardiac surgery and catheter or surgical AF ablation, especially if repeated, assessment of atrial contractility by transthoracic echocardiography should be performed before discontinuing OAC in patients who maintain sinus rhythm, confirmed by serial ECG or Holter monitorings.
\end{abstract}

Key words: atrial contractility, atrial fibrillation ablation, ECG, echocardiography, oral anticoagulation.

Abbreviation list:

AF: atrial fibrillation

CT: computed tomography

MRI: magnetic resonance imaging

OAC: oral anticoagulation

TIA: transient ischemic attack 


\section{Introduction}

Atrial fibrillation (AF) is a leading cause of thromboembolic events including strokes, transient ischemic attacks (TIA) [1] and silent cerebral infarctions [2]. AF ablation has become an effective strategy for symptomatic drug refractory AF [3,4]; however, indication for oral anticoagulation (OAC) to prevent thromboembolic events after a successful procedure is still debated.

This uncertainty is reflected by current guidelines. On the one hand, the 2010 Canadian atrial fibrillation guidelines suggested that "in the presence of sustained normal sinus rhythm, OAC should be discontinued only if the long-term risk of stroke is low (CHADS2 score <2)" [5]. On the other hand, the 2014 American and 2016 European atrial fibrillation guidelines provided no specific recommendation on long-term OAC use after successful atrial fibrillation ablation $[6,7]$.

In routine clinical practice the CHADS2, CHA2DS2VASc, and HAS-BLED scores are, in fact, adopted to guide decision regarding OAC management $[8,9]$.

Nevertheless, AF ablation has proved to reduce the risk for subsequent thromboembolic events to the level of patients with no history of AF indifferently of CHADS score and age [10]. In addition, several studies supported feasibility and safety of discontinuing OAC after successful ablation reporting low thromboembolic and reduced bleeding risks [11-15].

\section{Clinical series}

Given the aforementioned evidences, OAC discontinuation following a successful AF ablation may become challenging.

The first clinical example is based on a 67-year-old man. Since a rheumatic endocarditis complicated by peripheral embolization, he was on OAC. In 1984, he underwent mitral valve replacement with a biological prosthesis. In 1993 the mitral bio-prosthesis, due to rupture of a 
cusp, was replaced with a mechanical valve, newly urgently replaced by a bio-prosthesis, in 2007, because of paravalvular leak. In the meantime, in 2005, due to symptomatic persistent AF despite antiarrhythmic drugs, he underwent radiofrequency transcatheter AF ablation (pulmonary veins isolation, left atrium roof and mitral lines) and, three months later, because of atrio-ventricular blocks, a DDD pace-maker was implanted.

Eventually the patient was referred to our Department, in March 2013, for progressive dyspnea worsening. Echocardiography showed a well-functioning bio-prosthesis but a severe aortic regurgitation. In October he therefore underwent aortic and mitral (visualization of thrombotic material) valve replacement by bio-prosthesis, AF cryoablation by box lesion of the pulmonary veins, mitral isthmus line, and left appendage closure by suture of the ostium by 2 layers of polypropylene from inside the left atrium.

Following surgical intervention, he presented the ECG shown in Figure 1 (Panel A), apparently showing no atrial activity. At pace-maker interrogation, however, endocavitary markers showed sensed atrial $(1.5 \mathrm{mV})$ and ventricular $(15.6 \mathrm{mV})$ activity. Furthermore, atrial activity could be captured $(0.75 \mathrm{~V} @ 0.5 \mathrm{~ms})$, although not visible on the ECG, demonstrated by 1:1 atrioventricular conduction at the paced rate (Figure 1, Panel B). At transthoracic echocardiography the left atrium was severely dilated (max/min area $35,4 \mathrm{~cm}^{2} / 29 \mathrm{~cm}^{2}$; max/min volume $159 \mathrm{ml} / 119 \mathrm{ml}$ ). At transmitral Doppler the A wave was not present and at both lateral and septal tissue Doppler scans the a' wave was poorly perceivable (Figure 2). Overall, the total atrial function, including both reservoir and conduct function, was estimated around $25 \%$.

The second example is a 68-year-old woman. Since 1999 moderate aortic regurgitation and moderate-to-severe mitral regurgitation with preserved left ventricular ejection fraction were diagnosed. Due to increasing dyspnea with palpitations, in January 2012, she referred to our 
Department with rapid ventricular response $(150 \mathrm{bpm}) \mathrm{AF}$; on that occasion, besides other drugs, OAC was started. A month later an electric cardioversion was performed restoring sinus rhythm but symptomatic AF relapse promptly occurred. At echocardiography aortic regurgitation had progressed towards moderate-to-severe and severe mitral regurgitation was associated with a giant left atrium (area $40 \mathrm{~cm}^{2}$, volume $167 \mathrm{ml}$ ). A surgical intervention was therefore performed to replace the aortic valve with a biologic prosthesis, to repair the mitral valve and to ablate AF by cryoablation (pulmonary veins box, mitral isthmus line and a line towards the left appendage). After surgical intervention, the patient recovered well and presented the ECG showed in Figure 3; however, due to persistence of symptomatic AF paroxysms, in November 2012, an electrophysiologic mapping was performed and the reconnected right superior pulmonary vein isolated (Figure 4, Panel A). At transthoracic echocardiography the severely enlarged left atrium displayed a scarcely apparent a' wave on tissue Doppler (Figure 4, Panel B and C), highlighting the absence of mechanical contractility irrespective of the presence of a clearly evident $\mathrm{P}$ wave on ECG.

\section{Discussion}

The presented cases are outmost and any physician would recommend oral anticoagulation therapy based on clinical wisdom. It is known that AF ablation achieves better results in patients with normal or mildly enlarged left atrium [16], however it is not contraindicated also in patients with severely enlarged atrial volumes and decreased atrial contractility. The present clinical perspective points out how the ECG-guided approach, on which AF ablation success/failure is traditionally based, may be unreliable, suggesting that the assessment of the atrial anatomy and mechanical function by transthoracic echocardiography may add significant data for the decision process of continuing/discontinuing OAC after "successful" AF ablation. 
Nowadays many efforts are directed towards identifying simple prognostic parameters for the prediction of clinical outcomes after AF ablation. Within several attempts P-wave duration and morphology at surface ECG, reflecting the process of electrical and structural remodelling, have been suggested. Caldwell $\mathrm{J}$ et al. [17] showed that the presence of preexistent prolonged $\mathrm{P}$-wave duration $(>140 \mathrm{~ms})$ is associated with a higher risk of paroxysmal AF recurrence post ablation; whereas Park JK et al. [18] demonstrated that basal P-wave amplitude in lead $\mathrm{I}<0.1 \mathrm{mV}$ is an independent predictor of clinical recurrence of AF after ablation. On the other side, Kizilirmak F et al. [19] reported that patients with greater reduction in P wave amplitude on post-procedural ECG have lower recurrence rates. None of these esperiences, however, focused on the relation to subsequent thromboembolic risk. In our work, we suggest the hypothesis that after AF ablation the reduced P wave amplitude on ECG may correspond to a static LA and therefore to a higher risk of thromboembolism (as in the first clinical case); on the other hand, also when a clearly evident $\mathrm{P}$ wave is detectable on ECG after AF ablation, the LA contractility may not be adequate to prevent the formation of thrombi in a severely enlarged LA (as in the second clinical case). Thus, in addition to ECG analysis we suggest a direct evaluation of the structural LA remodelling by transthoracic echocardiography to better stratify the risk of systemic thromboembolism.

Other potential parameters have been suggested to predict the risk of systemic thromboembolism after AF ablation. For example Proietti M et al. [20] showed a high prevalence of abnormal carotid intima-media thickness in AF patients, particularly in case of the persistent/permanent form of the arrhythmia and carotid/vertebral atherosclerosis is per se associated with an increased risk of ischemic events (Paciaroni $\mathrm{M}$ et al [21]). In addition, TLAA isolation t needs to be taken into account: Fassini $\mathrm{G}$ et al. [22] affirmed, although in patients with non-valvular AF, that combined transcatheter AF ablation and LAA closure by 
percutaneous devices in patients high risk of stroke or contraindication to antithrombotic treatment, appears feasible and related to no clinical thromboembolic events in the follow-up. Indeed AF ablation patients have reported significantly lower risk of stroke compared to AF patients who do not undergo ablation [23] and this lower risk spans across all age groups [24] and all CHADS risk profiles [10]. In addition, reports have suggested that cessation of OAC after successful AF catheter ablation may result in a very low incidence of thromboembolic complications and in a reduced bleeding risk. Themistoclakis et al. [12] published the pooled outcomes of 3355 patients who were AF-free following ablation. OAC was discontinued in $2692(80.2 \%)$ patients after 3-6 months. The thromboembolic risk for patients in whom OAC was discontinued amounted to $0.03 \% /$ year, similar to that of $0.23 \% / y e a r$ for those patients onOAC. The major bleeding rate was, instead, $0.98 \% /$ year for patients on-OAC versus $0.02 \% /$ year for those in whom it was discontinued $(\mathrm{P}<0.0001)$. No embolic events were recorded in moderate-high risk patients $(\mathrm{CHADS} \geq 2$ ) off-OAC. Indeed these patients represented only a minority $(13 \%, n=347)$ of the entire cohort. Nevertheless, the Authors conclude that, also stratified by CHADS score, the risk-benefit ratio favoured OAC discontinuation even in patients with a moderate-high TE risk (CHADS scores 1 and $\geq 2$ ). Saad et al. [13] examined the long-term risk of thromboembolic events off-OAC in 327 patients with a successful AF catheter ablation. OAC was discontinued in $91 \%$ of the patients (switched to antiaggregants), none of whom had a thromboembolic event during follow-up. Three $(0.9 \%)$ hemorrhagic strokes were, instead, reported in patients receiving OAC. Eventually, Winkle et al. [14] evaluated 108 patients with a prior thromboembolic stroke/TIA who underwent successful AF ablation. OAC was discontinued in $55(50.9 \%)$ after a mean period of 7.3 months following the successful ablation procedure. No thromboembolic event occurred in the off-OAC group. 
Also after surgically managed atrial fibrillation ablation the number of thromboembolic events is low and appears unrelated to the CHADS2 [25], indicating that the decision to discontinue OAC after the Cox-Maze procedure should not be based solely on CHADS2 scores; rather, rhythm status, echocardiographic findings, and patient risk for bleeding should be considered [26].

Although surely representative, the aforementioned experiences report residual thromboembolic risk in off-OAC patients and relevant bleeding risk in on-OAC patients suggesting clear need for improvement.

As a consequence, despite AF ablation success defined on sinus rhythm restoration at ECG and maintenance by serial $24 \mathrm{~h}$ Holter recordings, additional data, especially on atrial contractility, may be needed.

Buber et al. [27] showed that a lack of LA mechanical contraction in sinus rhythm patients at three months after surgery was associated with a 5-fold increase in the risk for stroke during follow-up. Larger atria imposed a significant risk as well; LA volume index $\geq 33 \mathrm{ml} / \mathrm{m} 2$ was associated with a 3 -fold risk increase.

The origin of thrombi is predominantly from the left appendage, although the atrium is, especially this cohort of patients, a known source. The reduction in contractility occurs in the whole left atrium, then thrombus formation more likely will occur in anatomical sites more prone to blood stasis, as the left appendage. The assessment of left atrial contractility by means of transthoracic echocardiography therefore permits to infer the overall risk of thrombus formation in any atrial site. A more precise evaluation of the presence/absence of thrombi in the left appendage should needs instead be performed by transesophageal echocardiography.

Rossillo et al. [11] suggested atrial contractility assessment at transesophageal echocardiography amongst the criteria for continuing/discontinuing OAC following 
successful AF ablation. A transesophageal echocardiographic evaluation was performed in all patients at 3-month follow-up examination. On TEE, the presence of a higher peak velocity and a prolonged duration of early and end diastolic left appendage flow emerged as markers of improved contractility. In fact, no cardiovascular accidents, despite OAC discontinuing, occurred in patients with maintained atrial contractility at a follow-up of $15 \pm 7$ months. Eventually, also left atrial appendage (LAA) morphology may play a role in predicting thromboembolic risk. Even after controlling for comorbidities and CHADS score, patients presenting a linear, non-complex LAA morphology (e.g. Chicken Wing) at cardiac CT or MRI have shown to be less prone to embolic events [28]. Furthermore, LAA morphology complexity related to the burden of silent cerebral ischemia detected at cerebral MRI [29].

\section{Limitations}

The present perspective is just a point of departure, suggesting a new approach or "sensibility" in clinical practice. The presented clinical cases represent a minimal portion of the real world but, in our opinion, relevantly sustain the importance of the topic at least within patients undergoing AF ablation and valve surgery. In any case we are convinced the concept introduced by the present manuscript may have application in a wider framework.

\section{Conclusion}

The present clinical perspective suggests that, at least within patients with severely enlarged left atrium, previous cardiac surgery and catheter or surgical AF ablation, especially if repeated, assessment of atrial contractility by transthoracic echocardiography should be performed before discontinuing OAC also in patients who maintained sinus rhythm, confirmed by serial ECG or Holter monitorings. Further studies are warranted to investigate if this evaluation may be needed in all patients undergoing AF ablation and which 
echocardiographic parameters, including perhaps morphological markers, may be most representative with the aim to further improve management of AF patients. 


\section{Expert commentary}

Even if arrhythmia abolition represents the most important goal of AF ablation procedure, the preservation of postoperative atrial contractility proved to be an important point for stroke risk evaluation. It is reasonable to think that the absence of an efficacious mechanical atrial action recovery even if associated with sinus rhythm detection could favour intra-atrial thrombi formation and consequent thromboembolic systemic phenomena, thus making the decision to interrupt anticoagulant treatment unsafe.

In our opinion sinus rhythm and left atrial contractility recovery represent two inseparable arms of the same question, and both should be weighed up carefully with the objective of screening patients who could fully benefit from $\mathrm{OAC}$ continuation/discontinuation after $\mathrm{AF}$ ablation.

\section{Five-year view}

The population in the Western world is growing older and in 5 years atrial fibrillation will continue the trend towards increased prevalence.

Also the success of ablative strategies will continue to enhance with the progress of technological advances [30,31].

There is a need for improvement in patient stratification and personalization of care after atrial fibrillation ablation.

One area that could be further developed is stroke risk stratification, where our understanding of which patients should continue anticoagulants for stroke prevention could be further refined. A better understanding of that risk may make it possible to reduce in future the number of patients receiving anticoagulation after a successful AF ablation, lowering in this way the number of hemorrhagic complications. On the other hand, it will endorse the continuation of $\mathrm{OAC}$ in patients with a higher risk profile, preventing thromboembolic events. 
In this context, the echocardiographic assessment of the restoration of an efficient left atrial contractility will probably be included amongst the criteria for continuing/discontinuing OAC following successful AF ablation.

\section{Key issues}

- Atrial fibrillation (AF) ablation has become an effective strategy for symptomatic drug refractory AF.

- Indication for oral anticoagulation (OAC) to prevent thromboembolic events after a successful procedure is still debated.

- In routine clinical practice the CHADS2, CHA2DS2VASc, and HAS-BLED scores are adopted to guide decision regarding OAC management.

- Nevertheless, AF ablation has proved to reduce the risk for subsequent thromboembolic events to the level of patients with no history of AF indifferently of CHADS score and age.

- In addition, several studies supported feasibility and safety of discontinuing OAC after successful ablation reporting low thromboembolic and reduced bleeding risks.

- The assessment of atrial anatomy and mechanical function by transthoracic echocardiography may add significant data for the decision process of continuing/discontinuing OAC, in patients who maintain sinus rhythm, confirmed by serial ECG or Holter monitorings, after a successful AF ablation.

- A lack of left atrial mechanical contraction in sinus rhythm patients is associated with an increase in the risk for stroke during follow-up. Larger atria impose a significant risk as well.

- Our perspective is just a point of departure, suggesting a new "sensibility" in clinical practice. The presented clinical cases represent a minimal portion of the real world but, 
in our opinion, relevantly sustain the importance of the topic at least within patients undergoing AF ablation and valve surgery.Further studies are warranted to investigate if this evaluation may be needed in all patients undergoing AF ablation and which echocardiographic parameters, including perhaps morphological markers, may be most representative with the aim to further improve management of AF patients. 


\section{References}

[1] Wolf PA, Dawber TR, Thomas HE Jr, et al. Epidemiologic assessment of chronic atrial fibrillation and risk of stroke: the Framingham study. Neurology 1978; 28:973-977.

[2] Gaita F, Corsinovi L, Anselmino M, et al. Prevalence of silent cerebral ischemia in paroxysmal and persistent atrial fibrillation and correlation with cognitive function. J Am Coll Cardiol 2013; 62(21):1990-7.

[3] Calkins H, Kuck KH, Cappato R, et al. 2012 HRS/EHRA/ECAS expert consensus statement on catheter and surgical ablation of atrial fibrillation: recommendations for patient selection, procedural techniques, patient management and follow-up, definitions, endpoints, and research trial design. J Interv Card Electr 2012; 33:171-257.

[4] Cappato R, Calkins H, Chen SA, et al. Updated worldwide survey on the methods, efficacy, and safety of catheter ablation for human atrial fibrillation. Circ Arrhythm Electrophysiol 2010; 3(1):32-38.

[5] CCS Atrial Fibrillation Guidelines Committee. Verma A, Macle L, Cox J, et al. Canadian Cardiovascular Society atrial fibrillation guidelines 2010: catheter ablation for atrial fibrillation/atrial flutter. Can J Cardiol 2011; 27:60-66.

[6] January CT, Wann LS, Alpert JS, et al. 2014 AHA/ACC/HRS guideline for the management of patients with atrial fibrillation: a report of the American College of Cardiology/American Heart Association Task Force on practice guidelines and the Heart Rhythm Society. J Am Coll Cardiol 2014 Dec 2;64(21):e1-76. 
[7] Kirchhof P, Benussi S, Kotecha D, et al. 2016 ESC Guidelines for the management of atrial fibrillation developed in collaboration with EACTS. European Heart Journal (2016) 37, $2893-2962$.

[8] Gaita F, Sardi D, Battaglia A, et al. Incidence of cerebral thromboembolic events during long-term follow-up in patients treated with transcatheter ablation for atrial fibrillation. Europace (2014) 16, 980-986.

[9] Anselmino M, Garberoglio L, Gili S, et al. Left atrial appendage thrombi relate to easily accessible clinical parameters in patients undergoing atrial fibrillation transcatheter ablation: A multicenter study. Int J Cardiol. 2017 Apr 8. doi: 10.1016/j.ijcard.2017.04.014. [Epub ahead of print]

[10] Bunch TJ, May HT, Bair TL, et al. Atrial fibrillation ablation patients have long-term stroke rates similar to patients without atrial fibrillation regardless of CHADS2 score. Heart Rhythm 2013; 10:1272-1277.

[11] Rossillo A, Bonso A, Themistoclakis S, et al. Role of anticoagulation therapy after pulmonary vein antrum isolation for atrial fibrillation treatment. J Cardiovasc Med 2008; 9:51-55.

[12] Themistoclakis S, Corrado A, Marchlinski FE, et al. The Risk of Thromboembolism and Need for Oral Anticoagulation After Successful Atrial Fibrillation Ablation. J Am Coll Cardiol 2010; 55:735-43.

[13] Saad EB, d'Avila A, Costa IP, et al. Very Low Risk of Thromboembolic Events in Patients Undergoing Successful Catheter Ablation of Atrial Fibrillation With a CHADS 2 Score $\leq 3$ : A Long-Term Outcome Study. Circ Arrhythm Electrophysiol. 2011; 4:615-621. 
[14] Winkle RA, Mead RH, Engel G, et al. Discontinuing anticoagulation following successful atrial fibrillation ablation in patients with prior strokes. J Interv Card Electrophysiol 2013; 38(3):147-53.

[15] Gray RM, Nagendran M, Maruthappu M: Is it safe to stop anticoagulants after successful surgery for atrial fibrillation? Interactive CardioVascular and Thoracic Surgery 2011; 13:642649.

[16] Anselmino M, Scaglione M, Blandino A, et al. Pulmonary veins branching pattern, assessed by magnetic resonance, does not affect transcatheter atrial fibrillation ablation outcome. Acta Cardiol 2010; 65(6):665-74.

[17] Caldwell J, Koppikar S, Barake W, et al. Prolonged P-wave duration is associated with atrial fibrillation recurrence after successful pulmonary vein isolation for paroxysmal atrial fibrillation. J Interv Card Electrophysiol 2014;39:131 -8.

[18] Park JK, Park J, Uhm JS, et al. Low P-wave amplitude $(<0.1 \mathrm{mV})$ in lead I is associated with displaced inter-atrial conduction and clinical recurrence of paroxysmal atrial fibrillation after radiofrequency catheter ablation. Europace (2016) 18, 384-391.

[19] Kizilirmak F, Demir GG, Gokdeniz T, et al. Changes in Electrocardiographic P Wave Parameters after Cryoballoon Ablation and Their Association with Atrial Fibrillation Recurrence. Ann Noninvasive Electrocardiol 2016;21(6):580-587.

[20] Proietti M, Calvieri C, Malatino L, et al. Relationship between carotid intima-media thickness and non valvular atrial fibrillation type. Atherosclerosis. 2015 Feb;238(2):350-5. 
[21] Paciaroni M, Agnelli G, Ageno W, et al. Risk factors for cerebral ischemic events in patients with atrial fibrillation on warfarin for stroke prevention. Atherosclerosis. 2010 Oct;212(2):564-6.

[22] Fassini G, Conti S, Moltrasio M, et al. Concomitant cryoballoon ablation and percutaneous closure of left atrial appendage in patients with atrial fibrillation. Europace. 2016 Nov;18(11):1705-1710. Epub 2016 Jul 11.

[23] Noseworthy PA, Yao X, Deshmukh AJ, et al. Patterns of Anticoagulation Use and Cardioembolic Risk After Catheter Ablation for Atrial Fibrillation. J Am Heart Assoc. 2015;4:e002597.

[24] Blandino A, Toso E, Scaglione M, et al. Long-term efficacy and safety of two different rhythm control strategies in elderly patients with symptomatic persistent atrial fibrillation. $\mathrm{J}$ Cardiovasc Electrophysiol 2013; 24(7):731-8.

[25] Ad N, Henry L, Schlauch K, et al. The CHADS Score Role in Managing Anticoagulation After Surgical Ablation for Atrial Fibrillation. Ann Thorac Surg 2010;90:1257-62.

[26] Ad N, Henry L, Shuman DJ, et al. A More Specific Anticoagulation Regimen Is Required for Patients After the Cox-Maze Procedure. Ann Thorac Surg 2014;98:1331-8.

[27] Buber J, Luria D, Sternik L, et al. Left Atrial Contractile Function Following a Successful Modified Maze Procedure at Surgery and the Risk for Subsequent Thromboembolic Stroke. J Am Coll Cardiol 2011;58:1614-21.

[28] Di Biase L, Santangeli P, Anselmino M, et al. Does the left atrial appendage morphology correlate with the risk of stroke in patients with atrial fibrillation? Results from a multicenter study. J Am Coll Cardiol 2012; 60(6):531-8. 
[29] Anselmino M, Scaglione M, Di Biase L, Gili S, Santangeli P, Corsinovi L, et al: Left atrial appendage morphology and silent cerebral ischemia in patients with atrial fibrillation. Heart Rhythm 2014; 11(1):2-7.

[30] Bertaglia E, Fassini G, Anselmino M, et al. Comparison of ThermoCool ${ }^{\circledR}$ SF Catheter versus ThermoCool ${ }^{\circledR C}$ atheter in Achieving Persistent Electrical Isolation of Pulmonary Veins: a Pilot Study. J Cardiovasc Electrophysiol 2013; 24(3):269-73.

[31] Bisleri G, Glover B. Hybrid ablation for atrial fibrillation: current approaches and future directions. Curr Opin Cardiol. 2017 Jan;32(1):17-21. 


\section{Figure Legends}

Figure 1. ECG of the first clinical example. Panel $A$ : ECG of the first clinical example following the last surgical intervention, apparently showing no atrial activity; central box: pace-maker report with endocavitary markers showing sensed atrial and ventricular activity (not having the possibility to describe the axis on the ECG, the hypothesis that the sensed $\mathrm{p}$ wave could be due to retrograde conduction of a junctional rhythm cannot be excluded). Panel B: ECG during atrial pacing (as confirmed by pace-maker report in the central box) demonstrating, although no visible atrial activity on the ECG, a 1:1 atrioventricular conduction at the paced rate.

Figure 2. Echocardiographic evaluation of atrial contractility in the first clinical case (A: maximum area and maximum volume by Simpson's method in two-dimensional apical fourchamber view; B: minimal area and minimal volume by Simpson's method in twodimensional apical four-chamber view; C: pulsed-wave Doppler of transmitral flow showing no A wave; D: Doppler tissue image of lateral mitral annulus demonstrating a poorly perceivable a' wave).

Figure 3. ECG of the second clinical example following the last surgical intervention, showing a clearly evident $\mathrm{P}$ wave.

Figure 4. Left atrium voltage-map and echocardiographic assessment of the second clinical example. Panel $A$ : Posterior and lateral views of the left atrium voltage-map of the second clinical case showing low voltages (below $0.04 \mathrm{mV}$ ) on the entire posterior wall (due to previous cryosurgical ablation) and presence, instead, of higher voltages at the ostium of the right superior pulmonary vein resulted reconnected (target for subsequent isolation); Panel B: area and volume of the left atrium by Simpson's method in two-dimensional apical four-chamber view; Panel C: Doppler tissue image of lateral mitral annulus demonstrating a poorly apparent a' wave. 
Figure 1

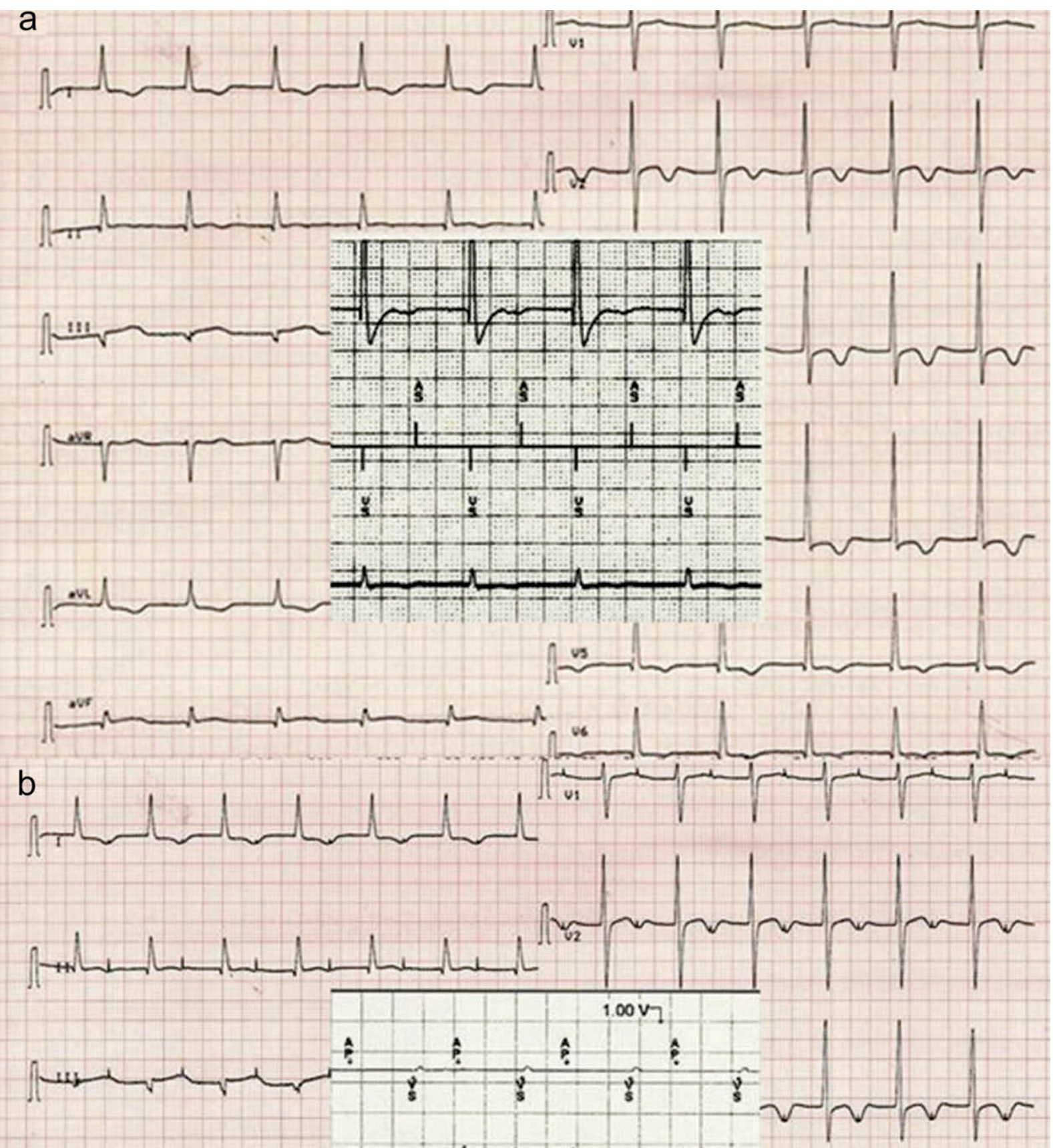

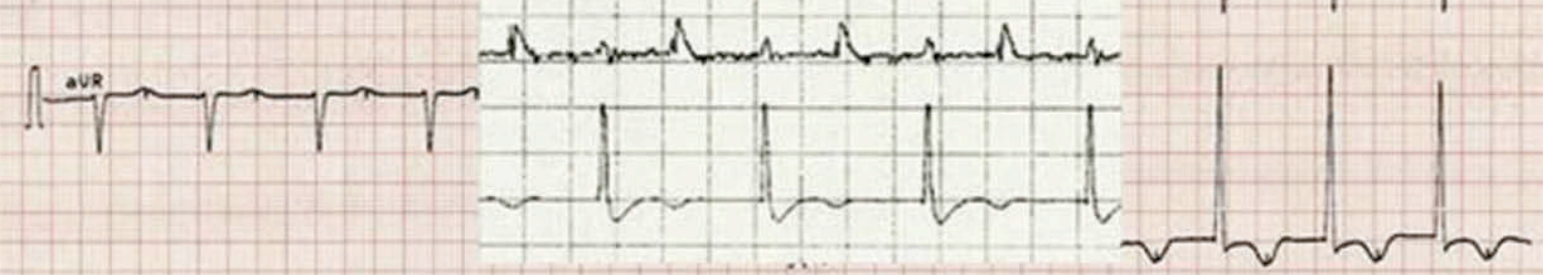

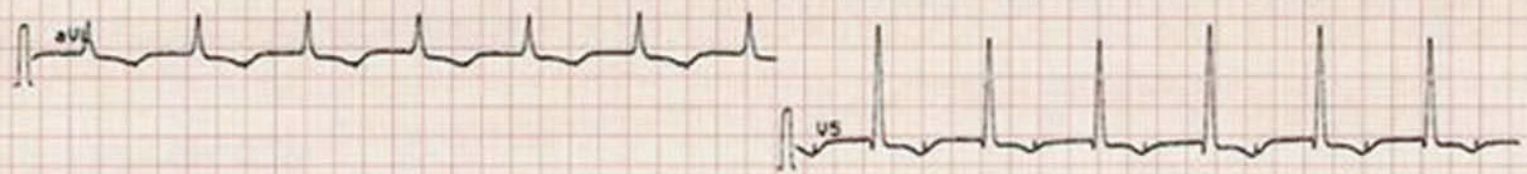

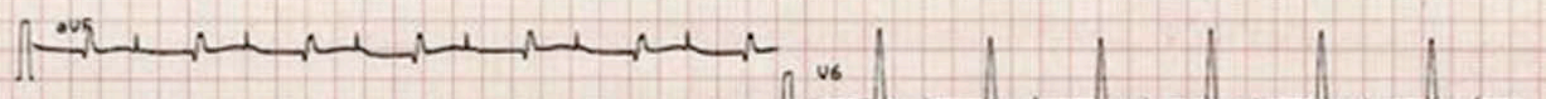


Figure 2

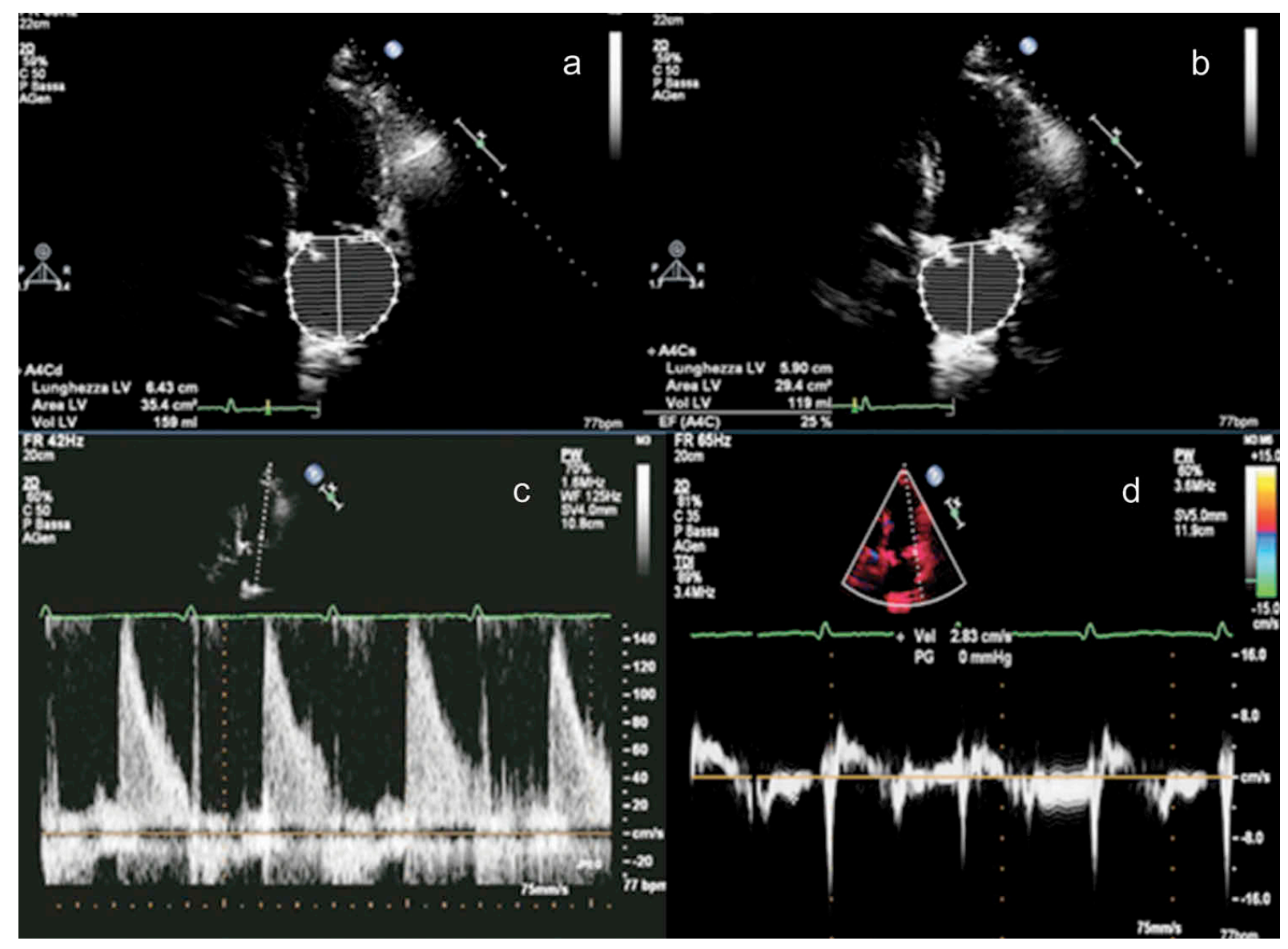




\section{Figure 3}

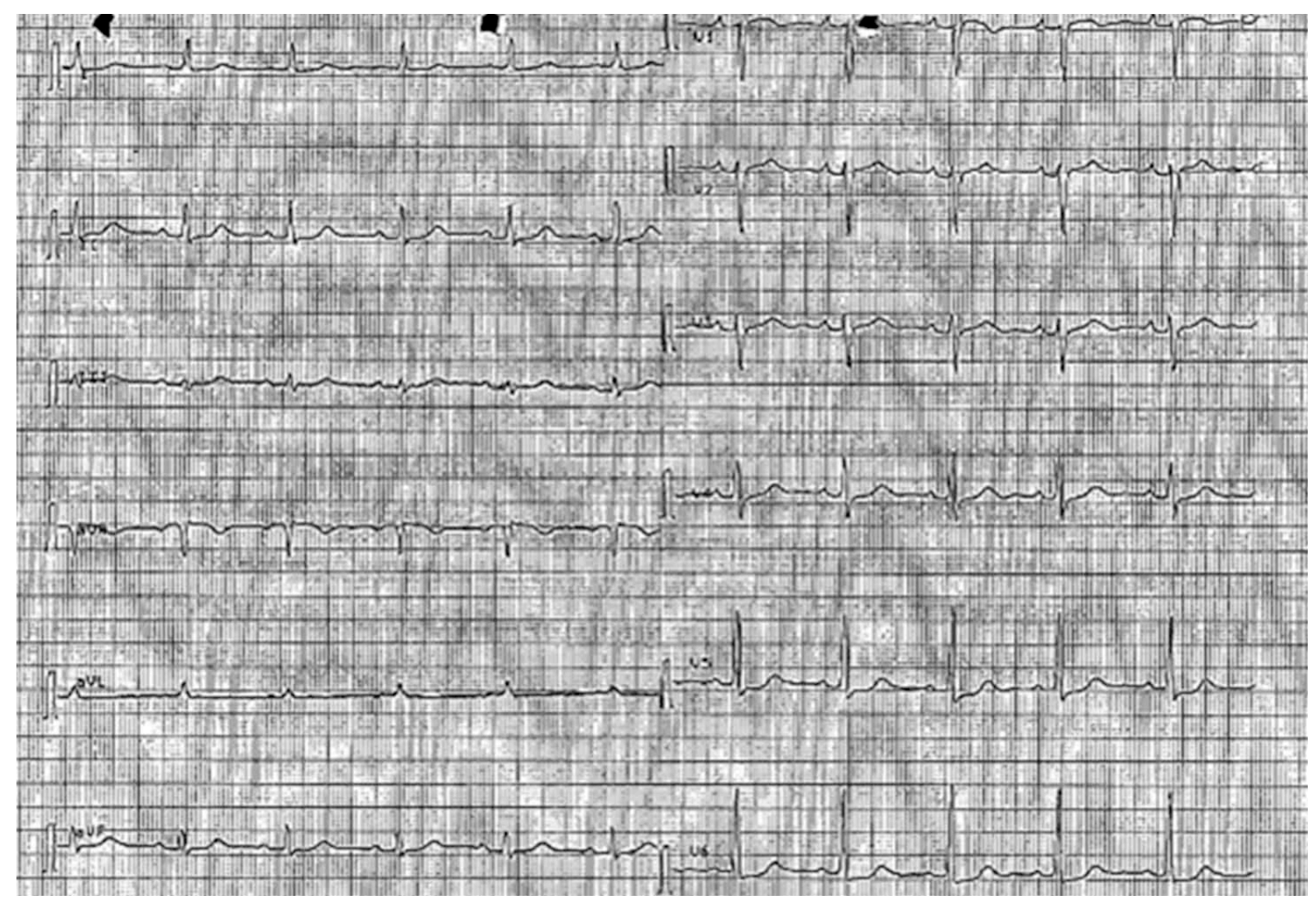


Figure 4

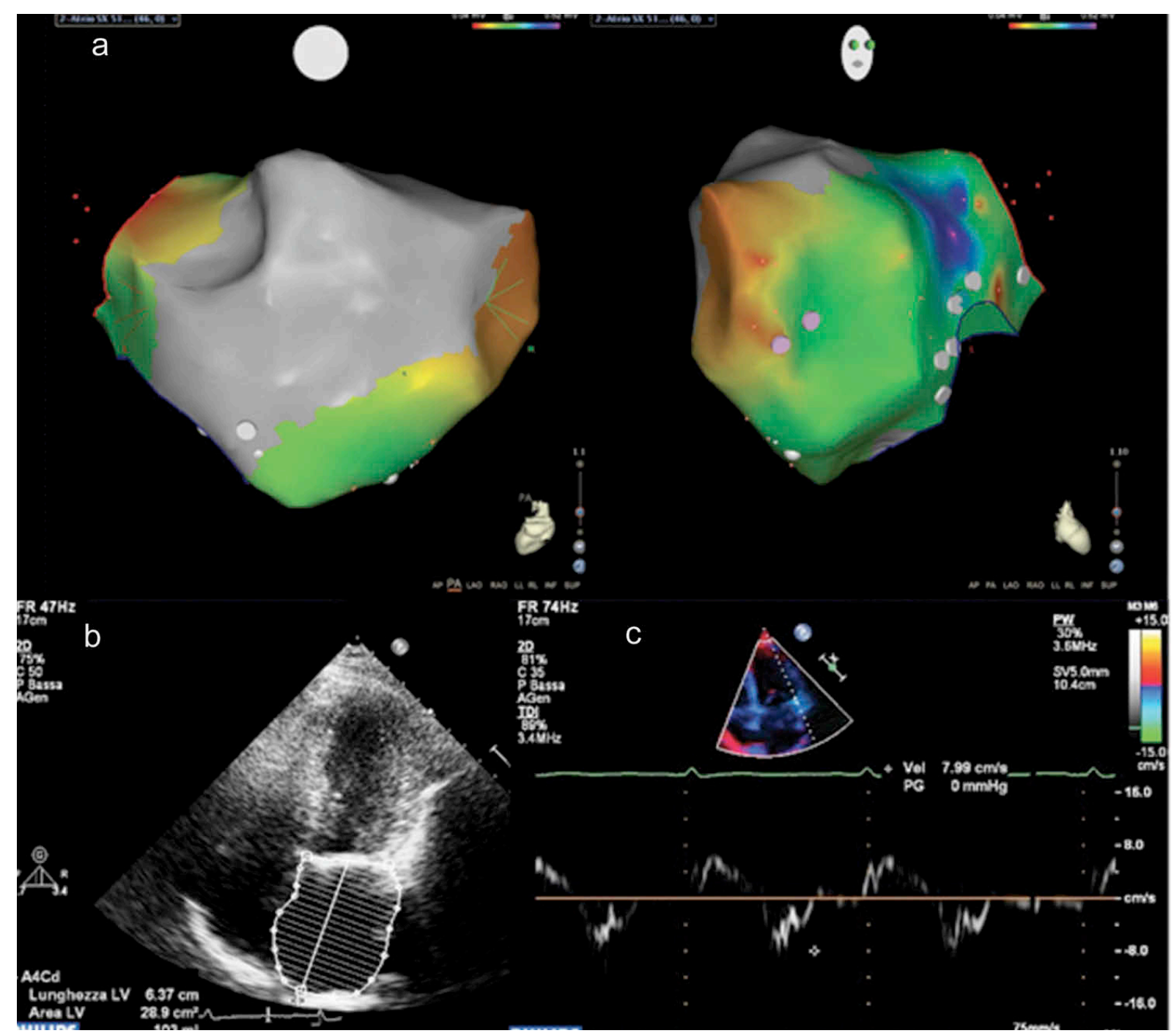

